\title{
From polarized to polarimetric Raman spectroscopy
}

\author{
A Frigout, M. Richert and R. Ossikovski \\ LPICM, Ecole Polytechnique, CNRS, 91128 Palaiseau, France
}

\begin{abstract}
Raman spectroscopy is widely used in many areas of research and technology such as chemical analysis and material properties characterization. "Classic" Raman spectroscopy traditionally neglecting the polarization-of-light aspect, polarized Raman spectroscopy has been developed to further explore the possibilities offered by Raman scattering. Polarized Raman spectroscopy is based on the control of the linear polarization states of the incident and scattered radiations. The next step for enlarging the scope of the Raman scattering technique is to perform a complete polarimetric control of the scattered, as well as incident light. In this contribution, we present the implementation and the calibration of a Stokes polarimeter within the Raman scattering setup. We performed the calibration of the detection path of the setup and noticed that it modifies substantially the polarization state of the scattered light. In particular, the system introduced effects such as depolarization, diattenuation and retardance that need to be estimated before proceeding with quantitative polarization analysis.
\end{abstract}

\section{Introduction}

During the last several decades Raman spectroscopy has become a reference tool for material and surface characterization [1]. For instance, this technique gives access to the nature of the chemical bonds [1] present in the substance under investigation. In order to get more information from a Raman spectroscopy experiment, a polarized Raman technique has been implemented and has provided important results in terms of stress measurements of strained materials [2]. By using this technique, we were able to measure the stress tensor in microelectronic structures such as biaxially strained $\mathrm{Si}$ and $\mathrm{SiGe}$ samples [3]. The natural continuation of such kind of studies is the experimental extension from polarized to polarimetric Raman spectroscopy allowing for Stokes vector and Mueller matrix polarization measurements. In what follows, we start by presenting the generic schematics of the polarimetric Raman experimental setup, consisting of the Raman spectrometer and the polarization control blocks. Next, we describe the calibration procedure and discuss the instrument accuracy.

\section{Experimental setup}

Figure 1 presents the principle schematics of a polarimetric Raman scattering setup. Its basic part is a Raman spectrometer (Labram HR800 from HORIBA Scientific) operated in normal (confocal) backscattering configuration.

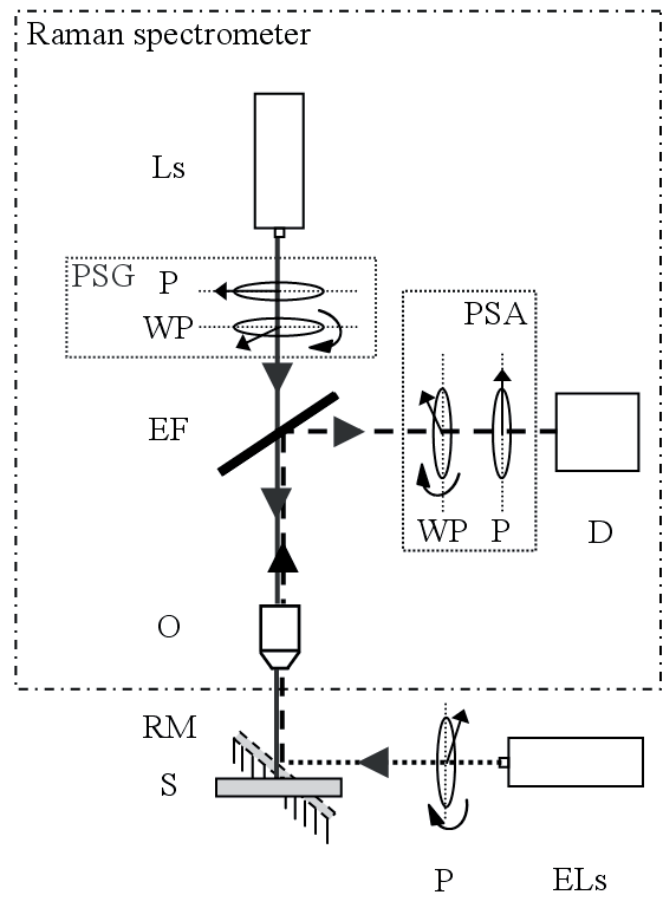

Figure 1. Principle schematics of the polarimetric Raman setup. Solid line: emission path; dashed line: detection path. Ls: laser source; P: polarizer; WP: quarter-wave plate; EF: edge filter; O: objective; D: detector (and grating); S: sample; PSG: polarization state generator; PSA: polarization state analyzer; ELs: external HeNe laser; RM: removable mirror.

As seen from the figure, in order to carry out measurements of the Stokes vector of the backscattered

This is an Open Access article distributed under the terms of the Creative Commons Attribution-Noncommercial License 3.0, which permits unrestricted use, distribution, and reproduction in any noncommercial medium, provided the original work is properly cited. 
light or, more generally, of the Mueller matrix of the sample, we introduced in front of the detector a polarization state analyser (PSA) consisting of a rotating quarter wave plate (02WMR003 from Melles Griot) followed by a fixed dichroic polarizer. A polarization state generator (PSG) comprising the same components, but with the polarizer placed before the retarder plate, was introduced after the laser source. These two polarization blocks make possible both the control of the incident polarization and the polarimetric analysis (measurement of the Stokes vector) of the scattered light. As a result of the combination of a Raman spectrometer and a PSG/PSA, one obtains a polarimetric Raman scattering setup.

The Stokes vector of the light passing through the PSG or PSA is obtained by rotating successively the retarder plate form $0^{\circ}$ to $180^{\circ}$ by steps of $5^{\circ}$ and recording each intensity spectrum. By performing Fourier analysis on the sixteen intensity measurements, one is able to determine the Stokes vector components [4].

\section{Calibration procedure}

One can readily see from figure 1 that the emission (laser) and detection (collection) light paths are entangled and cannot be calibrated separately within the optical configuration of the system. That is why, in a first step, we used an external laser source combined with a polarizer and a removable mirror (replacing the sample) to calibrate the detection path alone (represented by the dashed line in figure 1).

For this purpose, we measured the Stokes vector at the output of the detection path in the Rayleigh scattering regime. Linear input polarization states were generated by rotating a dichroic sheet polarizer (FPG007 from Melles Griot) at uniformly spaced azimuth values. The azimuth setting uncertainty of the polarizer was estimated to be $0.2^{\circ}$. The set of measured Stokes vectors is represented on the Poincaré sphere in figure 2, as a function of the linear incident state of polarization (varying between $0^{\circ}$ and $360^{\circ}$ by steps of $5^{\circ}$ ).

In absence of any polarization effects introduced by the system, all points in figure 2 should lie in the equator and be uniformly distributed, i.e. simply reproduce the input. However, one notices that the plane of the output polarization states is tilted with respect to the equatorial plane, which is certainly due to some retardance introduced by the system. One notices also that the polarization states are not uniformly distributed, a fact that can be readily attributed to the introduction of some diattenuation by the system. Finally, we can see that not all points lie on the surface of the sphere; this "effect" is directly related to a certain amount of depolarization that the optical path introduces.

In order to take into account the various polarization effects of the system (retardance, diattenuation and depolarization), we modelled the emission and detection paths as being both a dichroic retarder with a diattenuation $D$ and a retardance $\delta$ followed by a depolarizer with diagonal elements $[1,1, a, a](a \in[0,1]$ is the depolarization coefficient) and whose principal

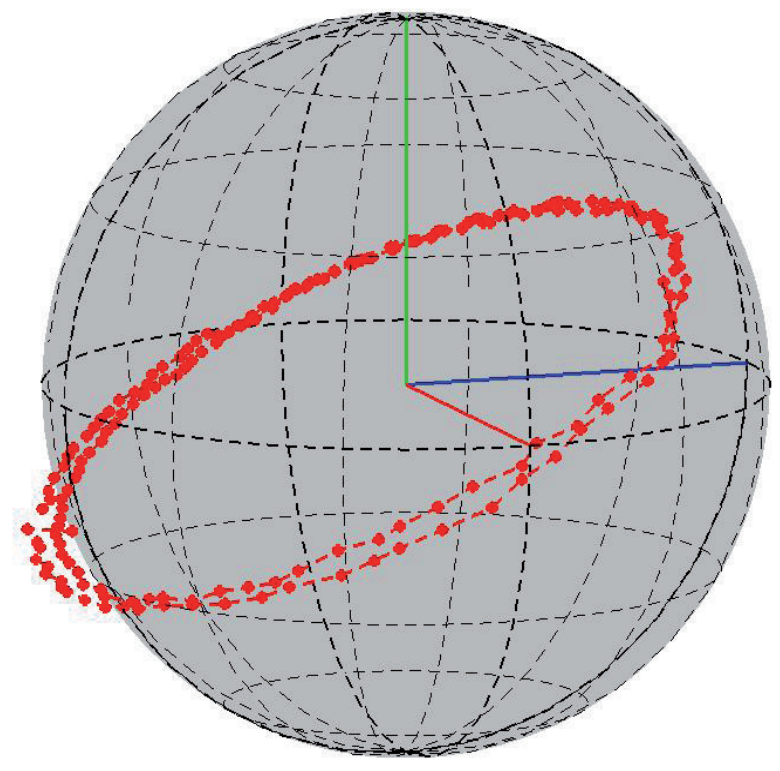

Figure 2. Poincaré sphere representation of the Stokes vector exiting the uncalibrated detection path obtained for uniformly varying linear polarization input states.

axes coincide with those of the dichroic retarder. Consequently, the Mueller matrices of these two polarization devices commute and the order of their multiplication is immaterial. Two more parameters used were the zero offsets of the axes of the quarter-wave plate and of the (fixed) polarizer of the PSA. All above listed parameters were determined (through fitting) in such a way so that the output Stokes vector, represented in figure 2, exhibit, after correction, the following properties:

- Average degree of polarization closest to 1.

- Average ellipticity closest to 0 .

- Uniform distribution of the polarization states on the equator

After calculation, we obtained for the dichroic retarder a diattenuation value $D=0.515$, a retardance value $\delta=28.66^{\circ}$ and a depolarization coefficient $a=$ 0.928 . The zero offsets of the retarder wave-plate and of the polarizer were found to be $-1.713^{\circ}$ and $0.881^{\circ}$ respectively. It should be also noted that the retardance value of the quarter-wave plate used was previously determined on a Mueller polarimeter (MM16 from HORIBA Scientific) to be $73.8^{\circ}$ at $633 \mathrm{~nm}$ (for a theoretical value of $78.2^{\circ}$ ).

\section{Measurement accuracy}

To assess the overall system performance after the calibration we performed the same series of Stokes vector measurements as the one in Section 3. The results are shown in figure 3 . One notices that now the polarization states indeed all lie in the equator and are uniformly distributed along it.

The mean deviation between the experimental and theoretical normalized Stokes vector elements equalled $[0.04,0.04,0.02]$, exhibiting values within the experimental uncertainty. Concerning the degree of 


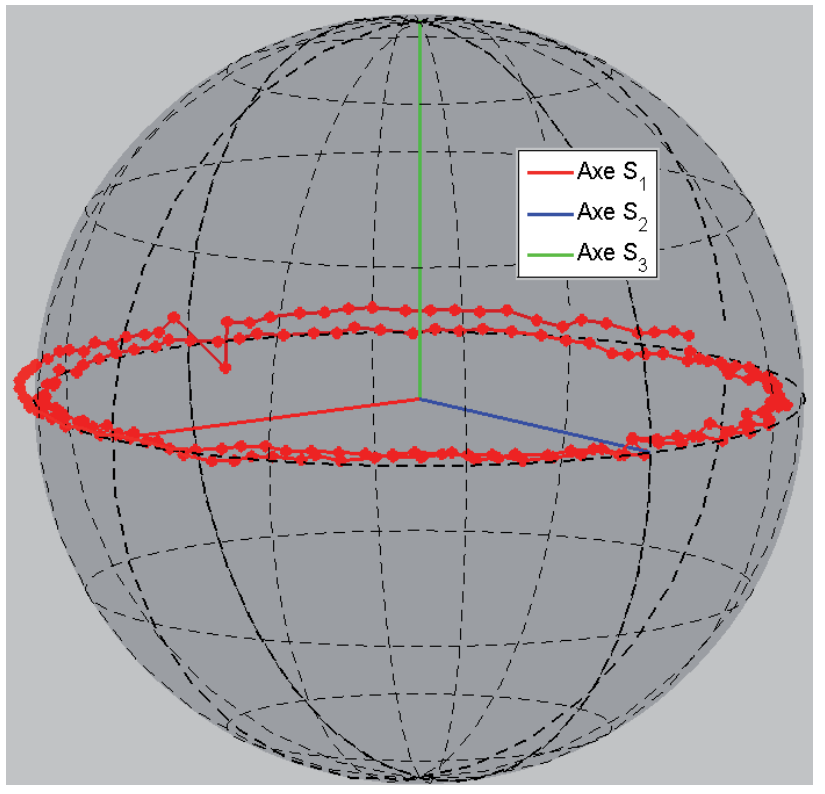

Figure 3. Poincaré sphere map of the polarization states exiting the detection path after calibration, obtained for uniformly varying linear polarization input states.

polarization, the standard deviation (with respect to unity) was about 0.04 . It can be also noticed from figure 2 that the two circles describing by the Stokes vector trajectories are not superimposed; this is to be most possibly attributed to an optical misalignment.

As a second test "sample" we used a dichroic sheet polarizer at a fixed azimuth followed by a quarter-wave plate measured at evenly spaced wave-plate azimuth values. Figure 4 represents the trajectory of the output Stokes vectors obtained, together with the theoretical curve (solid line) obtained from fitting to two parameters, the zero offset of the polarizer and the retardance of the wave-plate (values of $-1.2^{\circ}$ and $74.03^{\circ}$, respectively). For comparison, the wave-plate retardance, measured with the MM16 polarimeter, was found to be $74.3^{\circ}$, in very close agreement with the one given by the fit. (Note that this plate, together with the one used in the PSA, should ideally have the retardance of $78.2^{\circ}$ at $633 \mathrm{~nm}$, as mentioned in the end of Section 3.)

The standard deviation between the experimental and theoretical normalized Stokes vector components was about $[0.03,0.04,0.05]$. The small error level, of the order of the experimental uncertainty, confirms the correct calibration of the detection path of the Raman spectrometer.

\section{Conclusion}

The development of a polarimetric Raman experiment opens up the way to novel characterization opportunities such as the measurement of Stokes-Raman vectors or Mueller-Raman matrices. This study is only the first step on the way to a complete polarimetric Raman spectrometer. We have characterized polarimetrically the detection path of the system and shown that the spectrometer introduced significant polarization effects such as depolarization, retardance and diattenuation (with

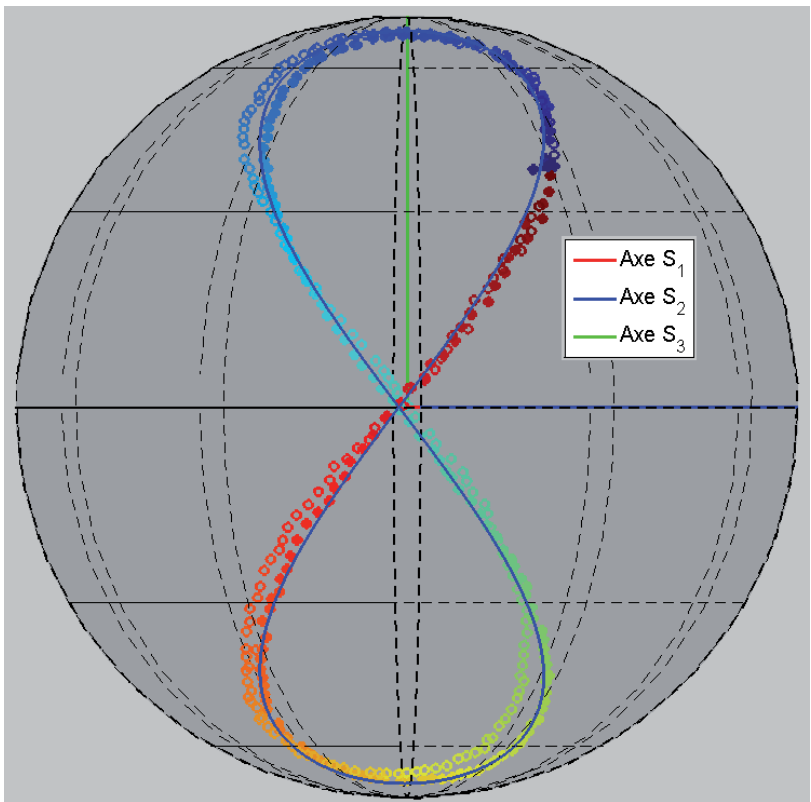

Figure 4. Poincaré sphere map of the polarization states exiting the detection path after calibration, for an elliptical polarization input state whose azimuth uniformly varies between $0^{\circ}$ and $360^{\circ}$. The solid line is the corresponding calculation for an elliptical state with a retardance of $74.03^{\circ}$.

respective values about $0.93,28.66^{\circ}$ and 0.515 ). After calibration, the instrument performance is quite satisfactory with an average accuracy of 0.04 on the Stokes vector components.

\section{References}

1. D.J. Gardiner, P.R. Graves (eds.) Practical Raman spectroscopy, (Springer Verlag, Berlin, 1989).

2. G.H. Loechelt, N.G. Cave, J. Menéndez, J. Appl. Phys. 86, 11 (1999).

3. R. Ossikovski, Q. Nguyen, G. Picardi, J. Schreiber, J. Appl. Phys. 103, 093525 (2008).

4 D.T. Pierce, A. Davies, J.A. Strocio, R.J. Celotta, Appl. Phys. A 66, S403 (1998). 